\title{
Application of the Kalman Filter to Estimate the State of an Aerobraking Maneuver
}

\author{
Willer Gomes dos Santos, Hélio Koiti Kuga, and Evandro Marconi Rocco
}

National Institute for Space Research (INPE), Avenida dos Astronautas, 1758, Jardim da Granja, 12227-010 São José dos Campos, SP, Brazil

Correspondence should be addressed to Willer Gomes dos Santos; willer.gomes@yahoo.com.br

Received 30 April 2013; Revised 11 June 2013; Accepted 17 June 2013

Academic Editor: Vivian Gomes

Copyright (C) 2013 Willer Gomes dos Santos et al. This is an open access article distributed under the Creative Commons Attribution License, which permits unrestricted use, distribution, and reproduction in any medium, provided the original work is properly cited.

\begin{abstract}
This paper presents a study about the application of a Kalman filter to estimate the position and velocity of a spacecraft in an aerobraking maneuver around the Earth. The cis-lunar aerobraking of the Hiten spacecraft as well as an aerobraking in a LEO orbit are simulated in this paper. The simulator developed considers a reference trajectory and a trajectory perturbed by external disturbances combined with nonidealities of sensors and actuators. It is able to operate in closed loop controlling the trajectory at each instant of time using a PID controller and propulsive jets. A Kalman filter utilizes the sensor data to estimate the state of the spacecraft. The estimation algorithms and propagation equations used in this process are presented. The U.S. Standard Atmosphere is adopted as the atmospheric model. The main results are compared with the case where the Kalman filter is not used. Therefore, it was possible to perform an analysis of the Kalman filter importance applied to an aerobraking maneuver.
\end{abstract}

\section{Introduction}

An orbital maneuver is described by the transferring of a satellite, from one orbit to another, through a changing in the velocity. To execute the maneuver, the spacecraft has to engage the thrusters or use the natural forces of the environment. The Hohmann transfer [1] and the Bielliptical transfer [2] are some alternatives to accomplish an orbital maneuver by propulsive means. If a velocity increment is added to the satellite velocity instantaneously, the maneuver is called impulsive maneuver [3-6]. The continuous thrust assumes that a finite thrust is applied by a time different from zero. The transfer orbit is a slow spiral outward under continuous thrust when the thrust is small compared to the gravitational force [7]. The theory of optimal low thrust orbital transfer has received a great deal of attention over the past years. There are many studies in the literature that consider a low thrust propulsion system [8-14] or an impulsive propulsion system [15-23]. Another kind of orbital transfer is the gravity-assisted maneuver (or swing-by) that consists in the use of the gravity of a planet or other celestial body to alter the path and speed of a spacecraft, typically in order to save propellant, time, and expense [24-33].

In 1961, Howard London presented the approach of using aerodynamic forces in order to change the trajectory of a spacecraft. This new technique became known as aeroassisted maneuvers [34]. This type of orbital transfer can be accomplished in several layers of the atmosphere. The altitude reached by the vehicle within the atmosphere is related to the mission purpose and the maximum thermal load supported by the vehicle structure. The main advantage of this type of maneuver is the fuel economy. As discussed by Walberg [34], many papers about aeroassisted orbital transfer have been made in recent decades and have shown that a significant reduction in fuel can be achieved using aeroassisted maneuvers instead of propulsive transfer [3538].

The transfer between two circular and coplanar orbits is widely used. The technique of using atmospheric drag to reduce the semimajor axis got known as aerobraking maneuver and was first used in 1991 by the spacecraft MUSESA (Space Engineering Spacecraft launched by MU rocket), 


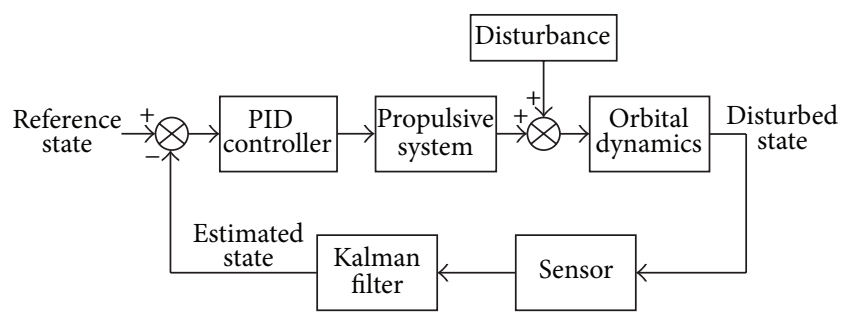

Figure 1: Basic diagram of the aeroassisted maneuver simulator.

which after its launch was renamed to Hiten. The launch was conducted by the Institute of Space and Astronautical Science of Japan (ISAS). The Hiten spacecraft passed through the Earth's atmosphere at an altitude of $125 \mathrm{~km}$ over the Pacific Ocean at a speed of $11 \mathrm{~km} / \mathrm{s}$. The experience leads to a decrease in the apogee altitude of about $8665 \mathrm{~km}$ [39]. In May 1993, an aerobraking maneuver was performed by the Magellan spacecraft in order to circularize its orbit around Venus. In 1997, the probe U.S. Mars Global Surveyor (MGS) has used its solar panels as "wings" to control its passage through the tenuous upper atmosphere of Mars to lower its apoapsis [40]. The future ESA Martian exploration program (ExoMars), with two missions to be launched in 2016 and in 2018, plans to use the aerobraking technique to circularize the initial high elliptical orbit, obtained after the Mars insertion [41].

In this paper, the Aeroassisted Spacecraft Maneuver Simulator (SAMS) was used with the implementation of the Kalman filter. The SAMS was based on an orbital maneuver simulator developed by Rocco [42] and used by Oliveira et al. [43]. Usually, it is used an open loop control operated from the ground for correction maneuvers and orbital transfers. However, in some missions, like drag-free (Gravity Probe B and Hipparcos), the feedback control is required. The SAMS considers a reference trajectory and a trajectory perturbed by external disturbances, including the aerodynamic effects, combined with nonidealities of sensors and actuators. It is able to operate in closed loop controlling the trajectory at each instant of time by a PID controller and propulsive jets. A study of how the orbital elements can be changed by an aeroassisted maneuver and how much fuel is saved is presented by Santos [44].

The Kalman filter is a tool that can estimate the variables of a wide range of processes and, from all the possible filters, it is the one that minimizes the variance of the estimation error. It is an estimator with real-time characteristics; that is, it provides estimates for the instant that the measurement is obtained [45]. The extended Kalman filter version [46] was used in this paper. In aerobraking maneuvers, the spacecraft operates close to the tolerable limits of the thermal loads [41] and a position error can cause the mission loss. Figure 1 shows a basic diagram about the running logic of the aeroassisted maneuver simulator.

In this paper, a spacecraft is assumed with a cubic body composed of two rectangular plates, called aerodynamic plates, placed in opposite sides of the vehicle body. The inclination angle of the plates with respect to the molecular flow is called of attack angle, whose value has been placed at 90 degrees to maximize the projected area and the drag force. In the second section, the mathematical modeling will be presented. The results and discussion are shown in the third section, while the conclusions are described in section four.

\section{Mathematical Modeling}

In an aerobraking maneuver, the spacecraft uses the drag of the upper layer of the atmosphere in order to decrease the spacecraft velocity to reach a target orbit. The aerobraking duration can take several months and it is characterized by a great number of passages by the atmosphere. After each passage by the atmospheric region, the reducing of the subsequent apogee occurs. When the spacecraft reaches the final apogee altitude, a new impulse is applied to the vehicle to remove it from the transfer orbit and to insert it into the target orbit. In order to control the thermal loads, propulsive jets are applied at the apogee to correct the decay of the perigee. This strategy is discussed in Walberg [34].

The spacecraft state is described by the coordinates $\mathbf{X}=\left[\begin{array}{llllll}X & Y & Z & \dot{X} & \dot{Y} & \dot{Z}\end{array}\right]$ measured in an inertial frame centered on the Earth. The Kepler equation provides the spacecraft trajectory. The sensor was modeled such that their measurements show a random error with zero mean and a nonzero variance. The dynamics model is composed by the gravitational acceleration and the acceleration of the atmospheric drag, as described by the following equation:

$$
\ddot{\mathbf{r}}=-\frac{\mu}{r^{3}} \mathbf{r}-\frac{1}{2} C_{D} \rho \frac{S}{M} V_{r} \mathbf{V}_{\mathbf{r}}+\mathbf{w},
$$

where $\mu$ is the gravitational parameter; $C_{D}$ is the drag coefficient; $\rho$ is the Earth's atmospheric density; $S$ is the projected area; $M$ is the spacecraft mass; $V_{r}$ is the velocity of the spacecraft relative to the atmosphere; $\mathbf{w}$ is the process noise (also known as dynamic noise). It was assumed that the normal of the surface area (projected area) is kept aligned with the spacecraft velocity relative to the atmosphere. The $\mathbf{w}$ parameter is modeled by a white process whose statistics is given by $\mathbf{w}=N(\mathbf{0}, \mathbf{Q})$, that is, zero mean and a covariance $\mathbf{Q}$. This noise is applied to the inertial position of the spacecraft.

The atmospheric model U.S. Standard Atmosphere [47] provides the value of the atmospheric density according to the altitude of the spacecraft, ranging from 0 to $2,000 \mathrm{~km}$. The velocity of the spacecraft relative to the atmosphere in the inertial system is calculated assuming that the atmosphere has the same rotation velocity of the Earth, as shown by the following equation:

$$
\mathbf{V}_{\mathbf{r}}=\dot{\mathbf{r}} \times \mathbf{r}=\left[\begin{array}{c}
\dot{x}+\omega y \\
\dot{y}-\omega x \\
\dot{z}
\end{array}\right]
$$

where $\mathbf{r}$ is the velocity vector relative to the inertial system and $\omega$ is the angular velocity of the Earth's rotation.

The Newtonian impact theory $[48,49]$ was used to compute the drag coefficient of the spacecraft, as described by the following equation:

$$
C_{D}=C_{p} \sin (\alpha)=2 \sin ^{3}(\alpha),
$$


where $C_{p}$ is the pressure coefficient, and $\alpha$ is the angle between the surface and the incident flow $\left(\alpha=90^{\circ}\right.$ in the study cases).

The deviation in the spacecraft trajectory was corrected by a PID controller. Most of the industrial controllers are Proportional-Integral-Derivative (PID) due to its low cost, robustness, and flexibility. The following equation shows the PID control law equation:

$$
c(t)=K_{P} \cdot e(t)+K_{I} \cdot \int e(t) d t+K_{D} \cdot \frac{d e(t)}{d t},
$$

where $K_{P}, K_{I}$, and $K_{D}$ are the proportional gain, integral gain, and derivative gain, respectively, and $e(t)$ is the position error in the trajectory. The observation model is given by the following equation:

$$
\mathbf{y}=\mathbf{h}(\mathbf{x})+\mathbf{v},
$$

where $\mathbf{y}$ is the measurement vector, $\mathbf{h}(\mathbf{x})$ is a nonlinear function of the state vector, and $\mathbf{v}$ is a vector of discrete white noise whose statistics is given by $\mathbf{v}=N(\mathbf{0}, \mathbf{R})$, that is, zero mean and a covariance $\mathbf{R}$. The measurement noise is due to the sensor reading of the spacecraft inertial position.

The extended Kalman filter generates some reference trajectories that are updated at each measurement processing. The filtering process consists of two stages: time-update and measurement-update. The following equation shows the time-update process:

$$
\begin{gathered}
\dot{\overline{\mathbf{x}}}=f(\overline{\mathbf{x}}), \\
\dot{\overline{\mathbf{P}}}=\mathbf{F} \overline{\mathbf{P}}+\overline{\mathbf{P}} \mathbf{F}^{T}+\mathbf{G Q G}^{T},
\end{gathered}
$$

where $\mathbf{F}$ is the Jacobian matrix of $f$ with respect to $\mathbf{x}, \mathbf{P}$ is the covariance matrix, and $\overline{\mathbf{x}}$ is the propagated state vector. The following equation describes the measurement-update process:

$$
\begin{gathered}
\mathbf{K}=\overline{\mathbf{P}} \mathbf{H}^{T}\left(\mathbf{H} \overline{\mathbf{P}} \mathbf{H}^{T}+\mathbf{R}\right)^{-1}, \\
\widehat{\mathbf{P}}=(\mathbf{I}-\mathbf{K} \mathbf{H}) \overline{\mathbf{P}}, \\
\widehat{\mathbf{x}}=\overline{\mathbf{x}}+\mathbf{K}[\mathbf{y}-\mathbf{h}(\overline{\mathbf{x}})],
\end{gathered}
$$

where $\mathbf{K}$ is the Kalman gain, $\mathbf{H}$ is the Jacobian matrix of $\mathbf{h}(\mathbf{x})$ with respect to $\mathbf{x}$ and it models how the observations are connected with the state, and $\widehat{\mathbf{x}}$ is the estimated state vector [45]. The estimated state is used by the PID controller to correct the trajectory error.

\section{Results and Discussion}

The results of an aerobraking maneuver simulation around the Earth using a Kalman filter to estimate the position and velocity of the spacecraft, at each instant of time, are presented in this section. Two cases are explored: the first shows an aerobraking performed in a LEO orbit, and the second study case presents the aerobraking simulation with the orbital elements of the Hiten spacecraft. At each step, the
TABLE 1: Initial conditions of the LEO orbit.

\begin{tabular}{lc}
\hline Description & Value \\
\hline Apogee altitude $(\mathrm{km})$ & 1000 \\
Perigee altitude $(\mathrm{km})$ & 120 \\
Eccentricity & 0.0634 \\
Inclination (degrees) & 1 \\
RAAN $^{1}$ (degrees) & 200 \\
Perigee argument (degrees) & 10 \\
Mean anomaly (degrees) & 180 \\
\hline
\end{tabular}

${ }^{1}$ Right ascension of the ascending node.

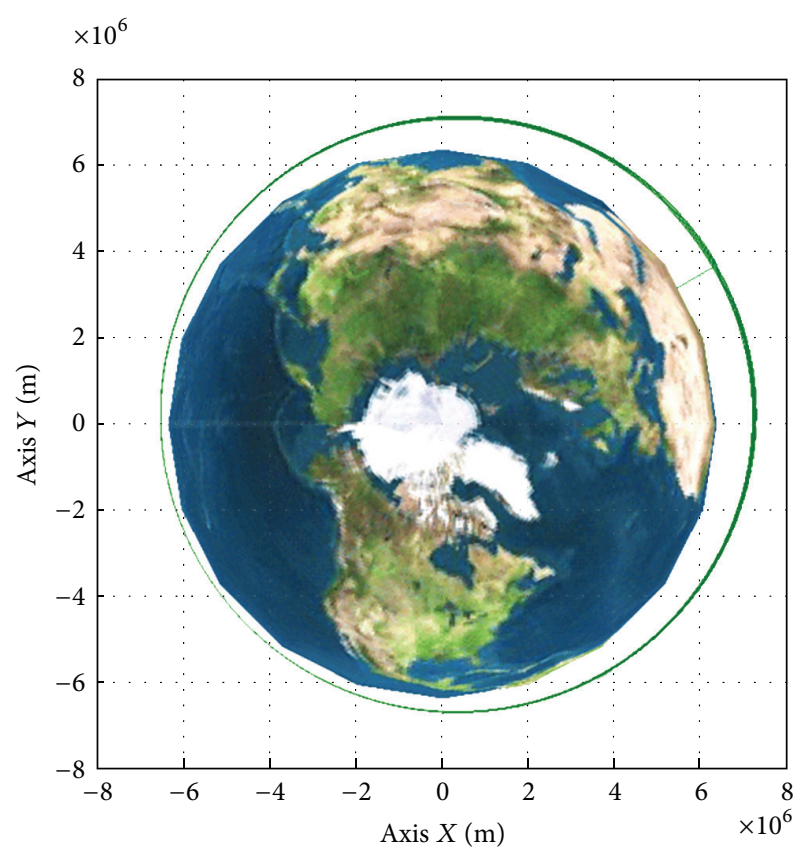

FIgURE 2: Spacecraft orbit in the $X Y$ plane.

PID controller sends a control signal to the propulsion system in order to correct the disturbed trajectory. The thrusters can apply a thrust of up to $20 \mathrm{~N}$ per second.

3.1. Aerobraking Maneuver in a LEO Orbit. In this first case, a spacecraft was assumed of $500 \mathrm{~kg}$ of mass, a cubic body of $1 \mathrm{~m}$ in each side and two aerodynamic plates of $2 \mathrm{~m}$ in length and $1 \mathrm{~m}$ in width. The step used in the simulation (sampling time) was of 1 second and it was accomplished up to eight hours of maneuvering. The propellant used was the liquid oxygen/liquid hydrogen, whose specific impulse is $460 \mathrm{~s}$. The main results are presented throughout this section. Table 1 shows the initial conditions of the orbit.

The spacecraft position is measured with an error of 0.5 meters (one standard deviation) and the dynamic noise has a standard deviation of 0.01 meters. The decrease of the apogee takes place in each aerobraking passage. Figure 2 shows the spacecraft orbit in the $X Y$ plane. In this graph, it is shown the perigee altitude inside the Earth's atmosphere and the decrease of the apogee altitude. The spacecraft altitude as function of time can be seen in Figure 3 . 


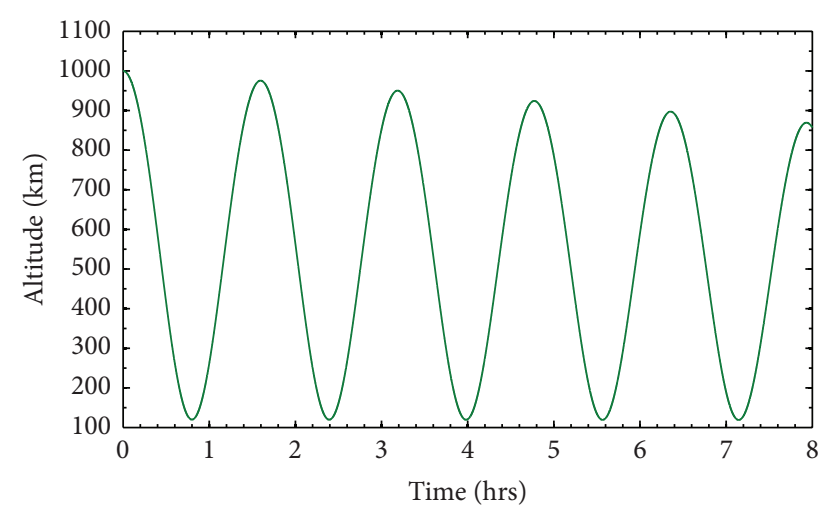

FIGURE 3: Spacecraft altitude as a function of time.

In both figures, it is possible to see the decrease of the apogee altitude whose final value is $869.30 \mathrm{~km}$, that is, a reduction of $130.70 \mathrm{~km}$. The perigee altitude remains around $120 \mathrm{~km}$. There was no change in the orbital inclination because the lift forces were not applied to the spacecraft. The estimated state is composed by the position and velocity of the spacecraft. Figure 4 shows the estimated position of the $X, Y$, and $Z$ coordinates while the estimated velocity is presented in Figure 5.

Figure 6 demonstrates the deviation of the spacecraft position, as a function of time, without the application of the Kalman filter, and in Figure 7 the same result can be seen but with the application of the Kalman filter. This divergence happens due to the measurement errors, causing a deviation between the reference trajectory and the disturbed trajectory. Throughout the maneuver, the trajectory control system acts to reduce this deviation. The deviation in the first case has reached values of approximately $2 \mathrm{~m}$, while, in the second case, the deviation was about $0.15 \mathrm{~m}$.

The deviation of the semimajor axis as a function of time presented can also be evaluated in Figure 8. The deviation in the first case (without the Kalman filter) has reached values of approximately $300 \mathrm{~m}$ while in the second case (with the Kalman filter) the deviation was about $35 \mathrm{~m}$.

The residue in the $X$ component of the position vector is presented in Figure 9. The other components $(Y$ and $Z$ ) of the position vector are similar and therefore were omitted. In the first graph, the results are presented for the complete maneuver (the time is shown in hours) while in the second graph, the residue are showed up to 3 minutes of maneuver in order to improve the visualization of the transient state (the time is shown in minutes). The blue line represents the error between the propagated position and the measured position; the green line represents the error between the propagated position and the Kalman filter's estimated position and the red line is 2 standard deviations. The estimated position error lies within about 0.5 meters while the measurement error occasionally presents spikes of up to 2 meters.

The final analysis of this study mentions the propellant consumption of the maneuvers without and with the Kalman filter. The results can be seen in Table 2 .
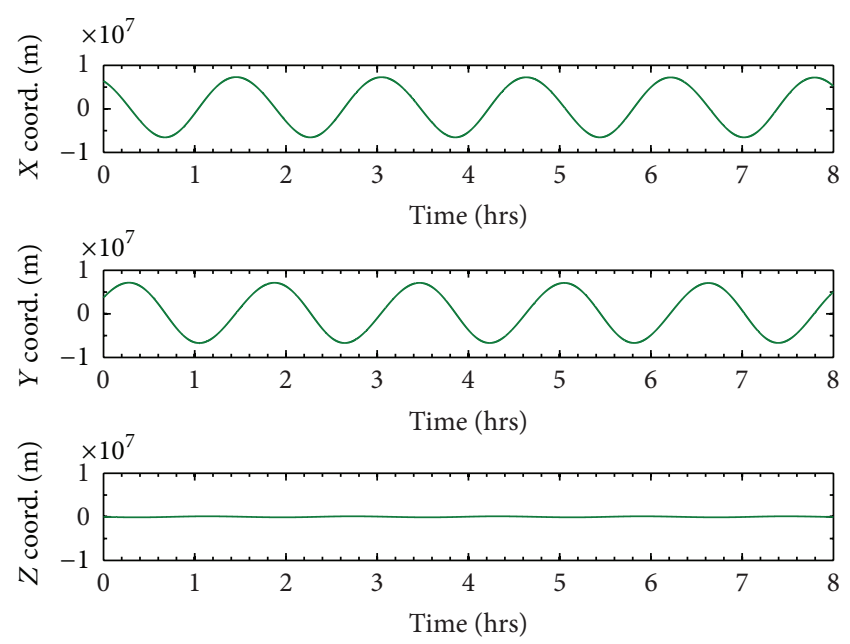

FIGURE 4: Estimated spacecraft position as a function of time.
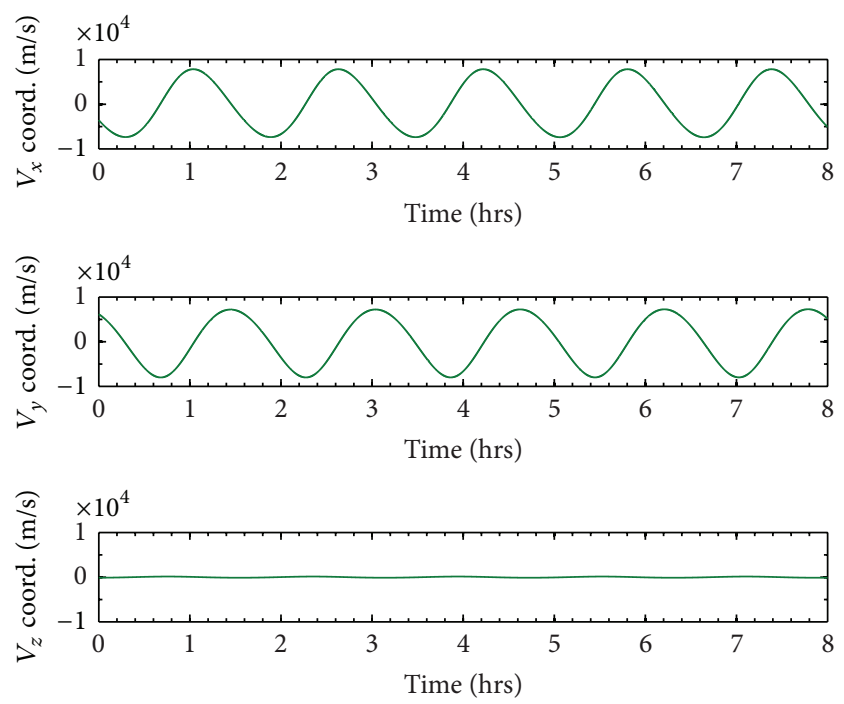

FIGURE 5: Estimated spacecraft velocity as a function of time.

As can be seen, the Kalman filter provided fuel savings of about $57 \mathrm{~kg}$ that represent almost $80 \%$ of reduction. The propellant was used to correct the trajectory between the reference state and the disturbed state. If the measurements accuracy was improved, then we have lower fuel consumption, as presented in Oliveira et al. [50].

3.2. Aerobraking Maneuver Using the Hiten Orbital Elements. The Hiten spacecraft successfully carried out its mission which included 10 lunar swingbys, insertion of a subsatellite into an orbit around the moon, 2 cis-lunar aerobraking experiments, excursion to the Lagrangian points (L4 and L5) of the Earth-Moon system, orbiting the Hiten spacecraft itself around the moon, and landing on the surface of the moon [39]. The spacecraft had a mass of $197 \mathrm{~kg}$ (at launch) including $42 \mathrm{~kg}$ of hydrazine fuel-adopted as the propellant for this simulation-and $12 \mathrm{~kg}$ for the lunar probe. The lunar probe was separated from the Hiten before the aerobraking 

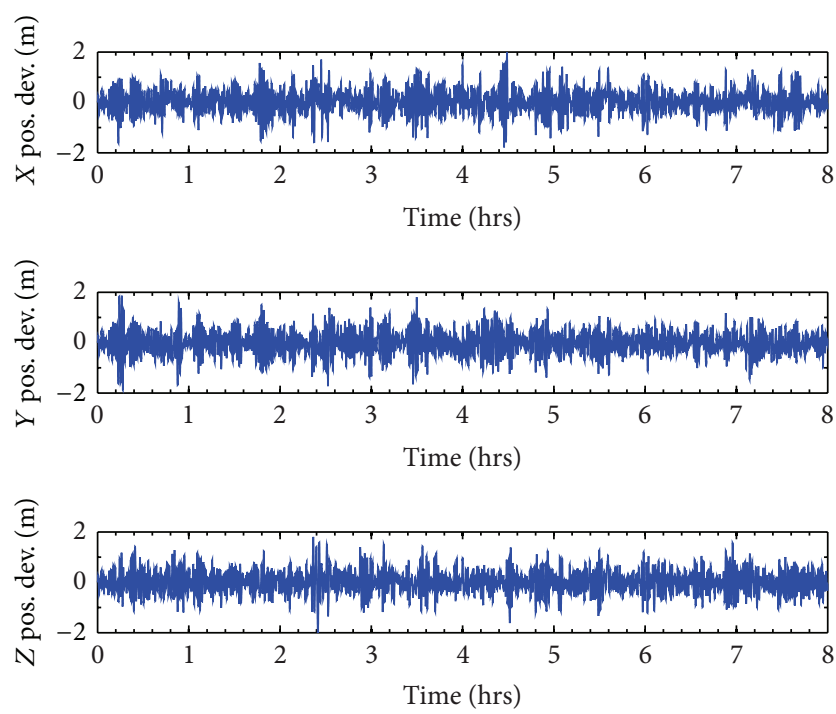

_ Without Kalman filter

FIGURE 6: Deviation in the spacecraft position, without the Kalman filter, as a function of time.
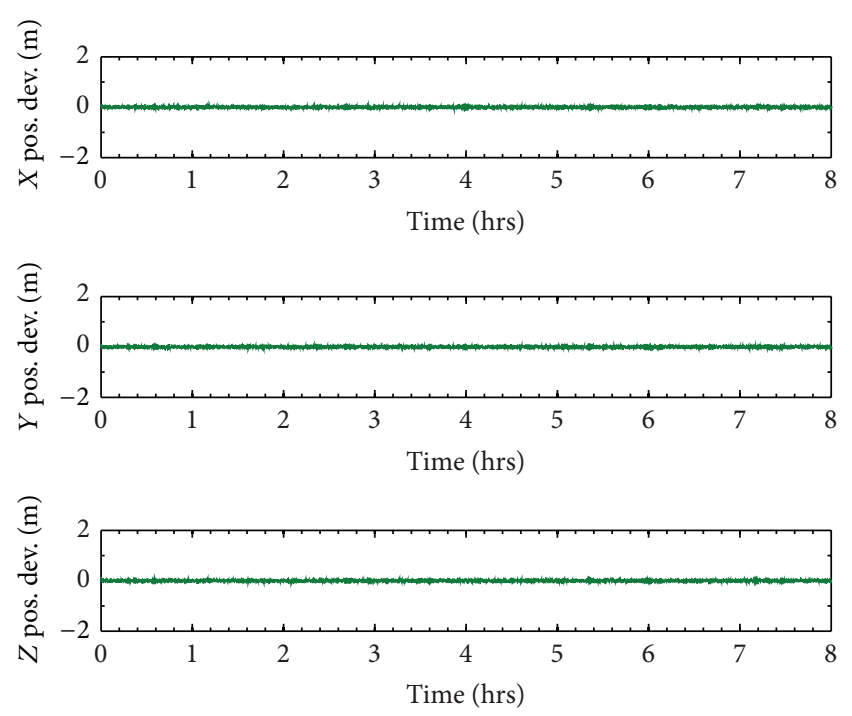

— With Kalman filter

FIgURE 7: Deviation in the spacecraft position, with the Kalman filter, as a function of time.

phase, so it was considered a spacecraft mass of $185 \mathrm{~kg}$. The vehicle was assumed to be a cubic body of $1 \mathrm{~m}$ in each side and two aerodynamic plates of $0.8 \mathrm{~m}$ in length and $0.4 \mathrm{~m}$ in width in order to approach the projected area of the Hiten spacecraft. The sampling time used in this simulation was 60 seconds. The spacecraft position is measured with an error of 0.1 meters (one standard deviation) and a dynamic noise was used with a standard deviation of 0.001 meters. Table 3 shows the initial conditions of the orbit.

The orbit in the $X Y$ plane is shown in Figure 10. As can be seen, the highly elliptical orbit has the apogee beyond

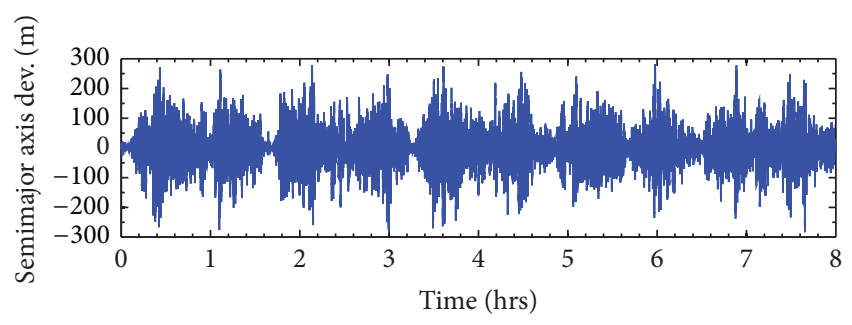

— Without Kalman filter

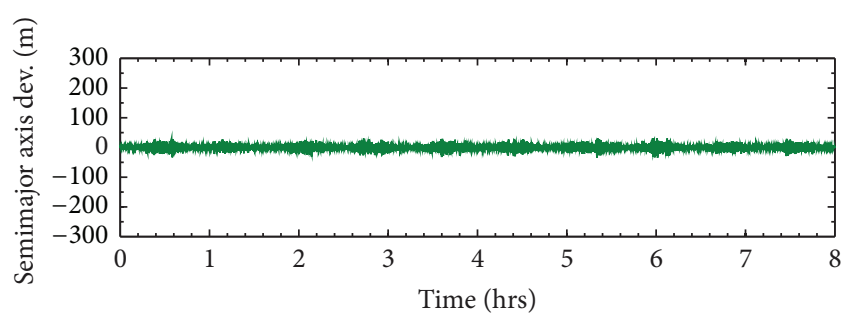

— With Kalman filter

Figure 8: Deviation in the semimajor axis (without and with the Kalman filter) as a function of time.
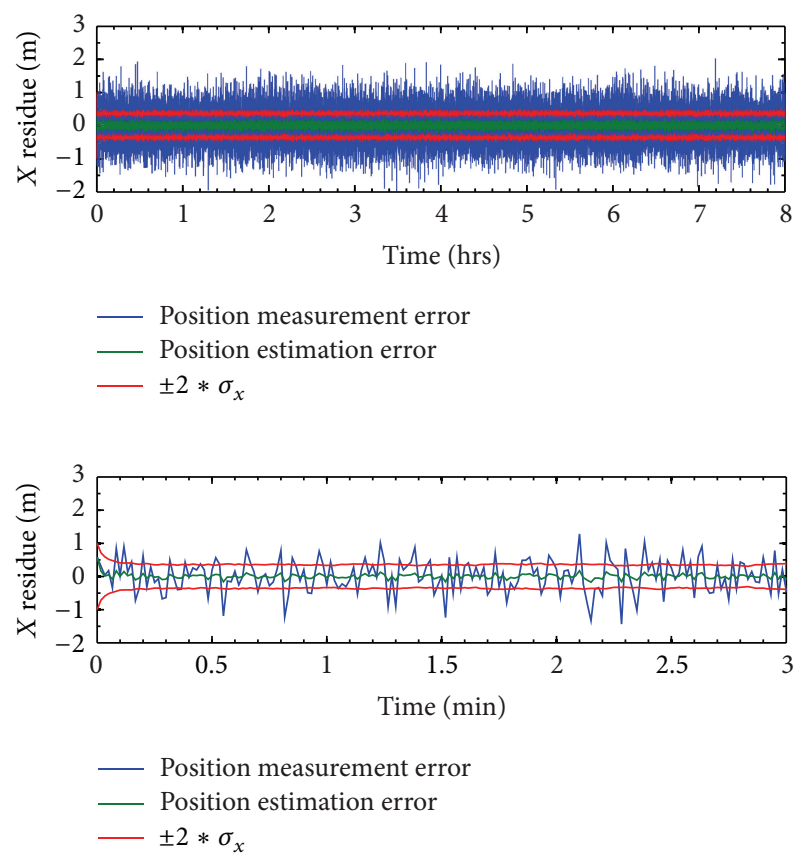

FIGURE 9: Residue in the $X$ component of the position vector.

TABLE 2: Propellant consumption analysis of the first case.

\begin{tabular}{lc}
\hline Maneuver & Propellant consumption $(\mathrm{kg})$ \\
\hline Without the Kalman filter & 71.54 \\
With the Kalman filter & 14.61 \\
\hline
\end{tabular}

the orbit of the moon, while the perigee is inside the Earth's atmosphere. The atmospheric drag decreased the subsequent apogee altitude to $416,439 \mathrm{~km}$, that is, a reduction of $8,561 \mathrm{~km}$ while the real mission had an apogee reduction of $8,665 \mathrm{~km}$. 


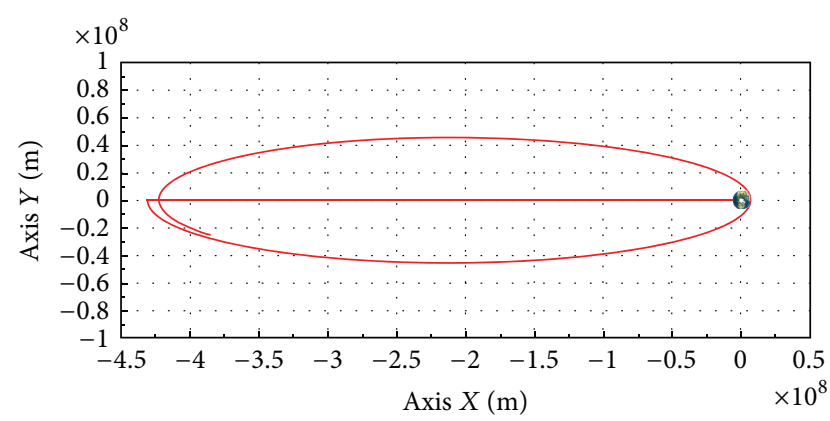

FIgURE 10: Hiten orbit in the $X Y$ plane.

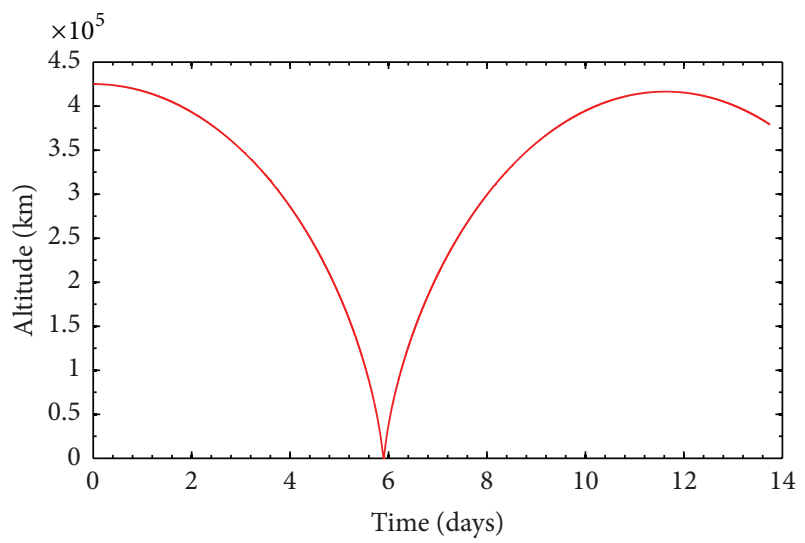

FIGURE 11: Spacecraft altitude as a function of time in days.

TABLE 3: Initial conditions of the Hiten orbit.

\begin{tabular}{lc}
\hline Description & Value \\
\hline Apogee altitude $(\mathrm{km})$ & 425,000 \\
Perigee altitude $(\mathrm{km})$ & 125 \\
Mean anomaly (degrees) & 180 \\
\hline
\end{tabular}

Figure 11 presents the spacecraft altitude as a function of time in units of days. The aerobraking passage happened near the sixth day and the orbital period is almost of 12 days.

The instant of the apogee reduction can be better visualized in Figure 12, which also shows the total drag suffered by the spacecraft as a function of time. It is possible to see that the higher drag force was almost $3.5 \mathrm{~N}$, whose value is related to the lowest altitude in the Earth's atmosphere.

Figure 13 shows the deviation of the semimajor axis as a function of time. The magnitude of the deviation increases when the spacecraft is suffering from the atmospheric drag effects. When the maneuver was performed without the Kalman filter, the divergence reached values of approximately $70,000 \mathrm{~km}$, while in the case where the Kalman filter was used the highest deviation was about $10,000 \mathrm{~km}$.

As discussed in the first study case, the Kalman filter can have its efficiency evaluated by the residue behavior, as presented in Figure 14. The other components of the position vector are similar and therefore were omitted. As defined before, the blue line represents the error between the propagated position and the measured position; the green
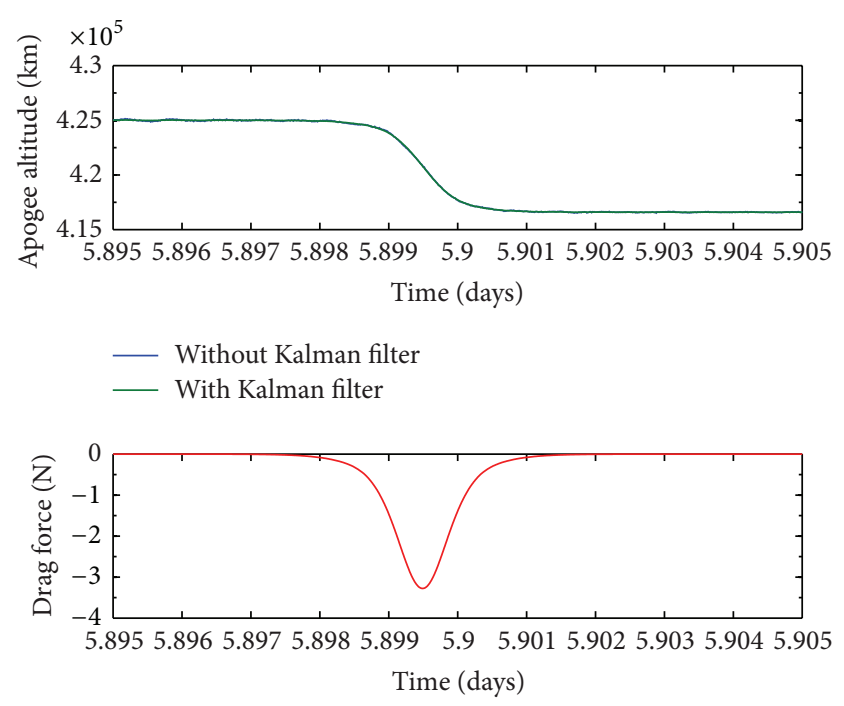

FIgURE 12: Apogee altitude and drag force as a function of time in days.

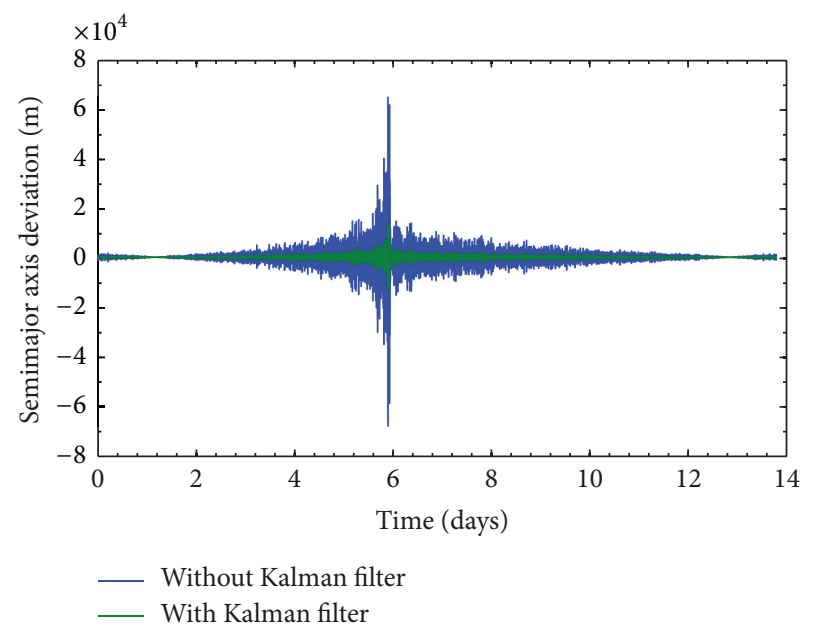

FIGURE 13: Semimajor axis deviation (without and with the Kalman filter) as a function of time in days.

line represents the error between the propagated position and the Kalman filter's estimated position and the red line is 2 standard deviations. In this case, the estimated position error lies within about 0.08 meters while the measurement error occasionally presents spikes of up to 0.4 meters.

The propellant consumption analysis, of the two types of maneuvers considered, is presented in Table 4. The Kalman filter provided a fuel saving of about $32 \mathrm{~kg}$. It is noteworthy that the Hiten spacecraft had $42 \mathrm{~kg}$ of fuel to realize all the objectives.

\section{Conclusions}

This paper presented a study of an aerobraking maneuver, around the Earth, using the Kalman filter to estimate the position and velocity of the spacecraft. A PID controller and 


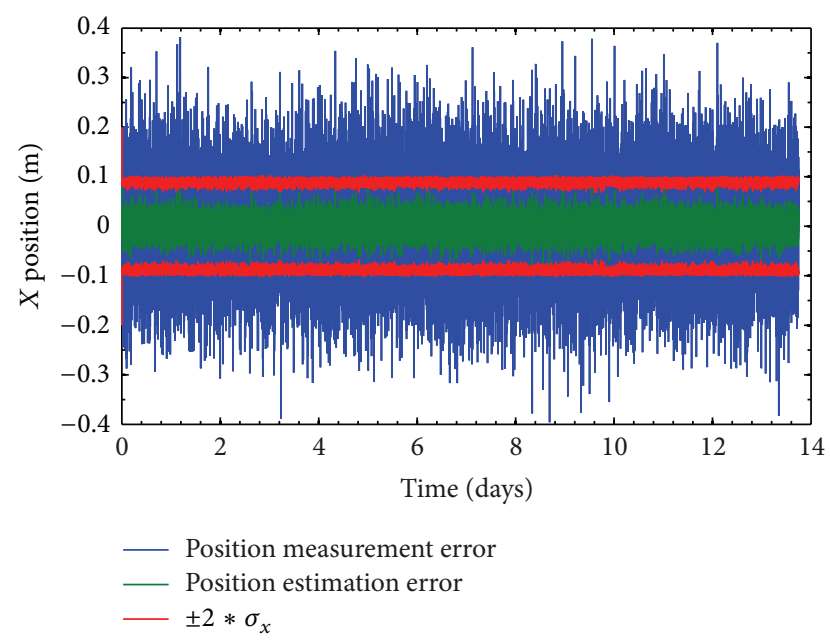

FIgURE 14: Residue in the $X$ component of the position vector as a function of time in days.

TABLE 4: Propellant consumption analysis of the second case.

\begin{tabular}{lc}
\hline Maneuver & Propellant consumption $(\mathrm{kg})$ \\
\hline Without the Kalman filter & 40.63 \\
With the Kalman filter & 8.96 \\
\hline
\end{tabular}

propulsive jets were used to correct the deviation between the reference trajectory and the disturbed trajectory. Two study cases were developed: an aerobraking in a LEO orbit was realized in the first case; the second case presented an aerobraking maneuver with the orbital elements similar to the Hiten spacecraft. The results with the application of the Kalman filter were compared to the case where the Kalman filter is not used. The results indicated that the Kalman filter decreased the position error and, also, provided a significant economy of fuel in both situations. Large errors in the spacecraft position can affect the control system performance. However, high-precision sensors have a high cost. Therefore, the mission design should consider the cost and benefit of each component taking into account the mission requirements.

\section{References}

[1] W. Hohmann, Die Erreichbarkeit Der Himmelskorper, Oldenbourg, Munique, Germany, 1925.

[2] R. F. Hoelker and R. Silber, "The bi-elliptic transfer between circular co-planar orbits," Tech Memo, Army Ballistic Missile Agency, Auburn, Ala, USA.

[3] F. W. Gobetz and J. R. Doll, "A survey of impulsive transfers," AIAA Journal, vol. 7, no. 5, pp. 801-834, 1969.

[4] D. F. Lawden, "Impulsive transfer between elliptical orbits," in Optimization Techniques, G. Leitmann, Ed., Academic, New York, NY, USA, 1962.

[5] A. F. B. A. Prado and R. A. Broucke, "Transfer orbits in restricted problem," Journal of Guidance Control and Dynamics, vol. 18, no. 3, pp. 593-598, 1995.
[6] E. M. Rocco, A. F. B. A. Prado, and M. L. O. Souza, "Threedimensional two-impulsive orbital maneuvers with time limit," WSEAS Transactions on Applied and Theoretical Mechanics, vol. 8, no. 1, pp. 2224-3429, 2013.

[7] T. N. Edelbaum, "Propulsion requirements for controllable satellites," ARS Jounal, vol. 31, no. 8, pp. 1079-1089, 1961.

[8] L. Casalino, G. Colasurdo, and D. Pastrone, "Optimal lowthrust scape trajectories using gravity assist," Journal of Guidance, Control, and Dynamics, vol. 22, no. 5, pp. 637-642, 1999.

[9] V. M. Gomes and A. F. B. A. Prado, "Low-thrust out-of-plane orbital station-keeping maneuvers for satellites," Mathematical Problems in Engineering, vol. 2012, Article ID 532708, 13 pages, 2012.

[10] B. L. Pierson and C. A. Kluever, "Three-stage approach to optimal low-thrust earth-moon trajectories," Journal of Guidance, Control, and Dynamics, vol. 17, no. 6, pp. 1275-1282, 1994.

[11] J. T. Betts and S. O. Erb, "Optimal low thrust trajectories to the moon," SIAM Journal on Applied Dynamical Systems, vol. 2, no. 2, pp. 144-171, 2003.

[12] Y.-J. Song, S.-Y. Park, K.-H. Choi, and E.-S. Sim, "A lunar cargo mission design strategy using variable low thrust," Advances in Space Research, vol. 43, no. 9, pp. 1391-1406, 2009.

[13] G. Mingotti, F. Topputo, and F. Bernelli-Zazzera, "Low-energy, low-thrust transfers to the Moon," Celestial Mechanics and Dynamical Astronomy, vol. 105, no. 1, pp. 61-74, 2009.

[14] S. A. Fazelzadeh and G. A. Varzandian, "Minimum-time earthmoon and moon-earth orbital maneuvers using time-domain finite element method," Acta Astronautica, vol. 66, no. 3-4, pp. 528-538, 2010.

[15] P. M. Lion and M. Handelsman, "Primer vector on fixed-time impulsive trajectories," AIAA Journaln, vol. 6, no. 1, pp. 127-132, 1968.

[16] E. M. Rocco, A. F. B. A. Prado, M. L. O. Souza, and J. E. Baldo, "Optimal bi-impulsive non-coplanar maneuvers using hyperbolic orbital transfer with time constraint," Journal of Aerospace Engineering, Sciences aNd Applications, vol. 1, no. 2, pp. 43-50, 2008.

[17] J. E. Prussing, "Optimal two- and three-impulse fixed-time rendezvous in the vicinity of a circular orbit," AIAA Journal, vol. 8, no. 7, pp. 1221-1228, 1970.

[18] K. Eckel, "Optimum transfer in a central force field with $\mathrm{n}$ impulses," Astronautica Acta, vol. 9, no. 5-6, pp. 302-324, 1963.

[19] K. G. Eckel, "Optimal impulsive transfer with time constraint," Acta Astronautica, vol. 9, no. 3, pp. 139-146, 1982.

[20] J. E. Prussing and J.-H. Chiu, "Optimal multiple-impulse timefixed rendezvous between circular orbits," Journal of Guidance, Control, and Dynamics, vol. 9, no. 1, pp. 17-22, 1986.

[21] L. Ting, "Optimum orbital transfer by several impulses," Astronautica Acta, vol. 6, no. 5, pp. 256-265, 1960.

[22] A. F. B. A. Prado and R. Broucke, "Transfer orbits in the Earthmoon system using a regularized model," Journal of Guidance, Control, and Dynamics, vol. 19, no. 4, pp. 929-933, 1996.

[23] N. Assadian and S. H. Pourtakdoust, "Multiobjective genetic optimization of Earth-Moon trajectories in the restricted fourbody problem," Advances in Space Research, vol. 45, no. 3, pp. 398-409, 2010

[24] J. M. Walton, C. Marchal, and R. D. Culp, "Synthesis of the types of optimal transfers between hyperbolic asymptotes," AIAA Journal, vol. 13, no. 8, pp. 980-988, 1975.

[25] G. A. Flandro, "Fast reconnaissance missions to the outer solar system utilizing energy derived from the gravitational field of Jupiter," Astronautica Acta, vol. 12, no. 4, pp. 329-337, 1966. 
[26] A. F. B. A. Prado, "Powered swingby," Journal of Guidance, Control, and Dynamics, vol. 19, no. 5, pp. 1142-1147, 1996.

[27] A. F. B. A. Prado and R. Broucke, "Effects of atmospheric drag in swing-by trajectory," Acta Astronautica, vol. 36, no. 6, pp. 285290, 1995.

[28] A. F. B. A. Prado, "Close-approach trajectories in the elliptic restricted problem," Journal of Guidance, Control, and Dynamics, vol. 20, no. 4, pp. 797-802, 1997.

[29] D. Dunham and S. Davis, "Optimization of a multiple lunarswingby trajectory sequence," Journal of Astronautical Sciencesn, vol. 33, no. 3, pp. 275-288, 1985.

[30] R. W. Farguhar and D. W. Dunham, "A new trajectory concept for exploring the earth's geomagnetic tail," Journal of Guidance Control, vol. 4, no. 2, pp. 192-196, 1981.

[31] D. Dunham and S. Davis, "Optimization of a multiple lunar swing by trajectory sequence," Journal of Astronautical Sciences, vol. 33, no. 3, pp. 275-288, 1985.

[32] A. F. B. A. Prado, "A comparison of the "patched-conics approach" and the restricted problem for swing-bys," Advances in Space Research, vol. 40, no. 1, pp. 113-117, 2007.

[33] A. F. B. A. Prado, "Orbital control of a satellite using the gravity of the moon," Journal of the Brazilian Society of Mechanical Sciences and Engineering, vol. 28, no. 1, pp. 105-110, 2006.

[34] G. D. Walberg, "A survey of aeroassisted orbit transfer," Journal of Spacecraft and Rockets, vol. 22, no. 1, pp. 3-18, 1985.

[35] W. Schulz, A. F. B. A. Prado, and R. V. De Moraes, "An analytical and numerical study of plane change maneuvers using aerodynamic force," Journal of the Astronautical Sciences, vol. 50, no. 3, pp. 289-303, 2003.

[36] A. Miele, "Recent advances in the optimization and guidance of aeroassisted orbital transfers," Acta Astronautican, vol. 38, no. 10, pp. 747-768, 1996.

[37] N. X. Vinh and J. M. Hanson, "Optimal aeroassisted return from high earth orbit with plane change," Acta Astronautica, vol. 12, no. 01, pp. 11-25, 1985.

[38] D. S. Naidu, J. L. Hibey, and C. D. Charalambous, "Fuel-optimal trajectories for aeroassisted coplanar orbital transfer problem," IEEE Transactions on Aerospace and Electronic Systems, vol. 26, no. 2, pp. 374-381, 1990.

[39] K. T. Uesungi, "Results of the MUSES-A 'HITEN' mission," Advanced Space Researchn, vol. 18, no. 11, pp. 1169-1172, 1996.

[40] R. E. Gladden, "Mars reconnaissance orbiter: aerobraking sequencing operations and lessons learned," Space Operations Communicatorn, vol. 06, no. 01, 2009.

[41] R. G. Jané and V. Companys, "Operational approach for the exomars aerobraking," in Proceedings of the 23rd Inernational Symposium on Space Flight Dynamics (ISSFD '12), Pesadena, Calif, USA, 2012.

[42] E. M. Rocco, Perturbed Orbital Motion With A PID Control System For the Trajectory, XIV Colóquio Brasileiro de Dinâmica Orbital, Águas de Lindóia, Brazil, 2008.

[43] T. C. Oliveira, E. M. Rocco, J. L. Ferreira, and A. F. B. A. Prado, "Minimum fuel low-thrust for satellites using a permanent magnet hall thruster," Mathematical Problems in Engineering, vol. 2013, 2013.

[44] W. G. Santos, Simulação de Manobras Aeroassistidas de um Veículo Espacial Controlado por Placas Aerodinâmicas Reguláveis e Sistema Propulsivo [M.S. Dissertation], National Institute for Space Research (INPE), São José dos Campos, Brazil, 2011.
[45] P. S. Maybeck, Stochastic Models, Estimation and Control, vol. 1, 1979.

[46] A. H. Jazwinski, Stochastic Processes and Filtering Theory, Academic Press, New York, NY, USA, 1970.

[47] A. Tewari, Atmospheric and Space Flight Dynamics: Modeling and Simulation With MATLAB? and Simulink, New York, NY, USA, 1 edition, 2007.

[48] F. J. Regan and S. T. Anandakrishnan, Dynamics of Atmosphere Re-entry, American Institute of Aeronautics and Astronautics, Washington, DC, USA, 1 edition, 1993.

[49] N. X. Vinh, A. Busemann, and R. D. Culp, Hypersonic and Planetary Entry Flight Mechanics, Elsevier, Amsterdam, The Netherlands, 1970.

[50] T. C. Oliveira, E. M. Rocco, and H. K. Kuga, "Análise de manobras orbitais de baixo empuxo usando o filtro de Kalman," in $10^{a}$ Conferência Brasileira de Dinâmica, Controle e Aplicações (DINCON), Águas de Lindóia, Brazil, 2011. 


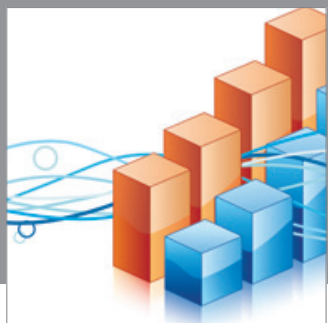

Advances in

Operations Research

mansans

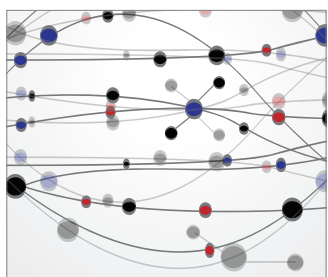

The Scientific World Journal
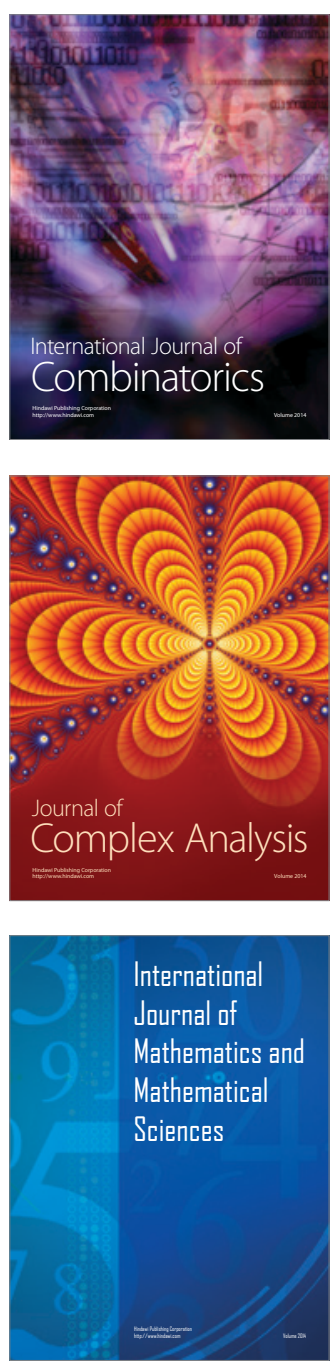
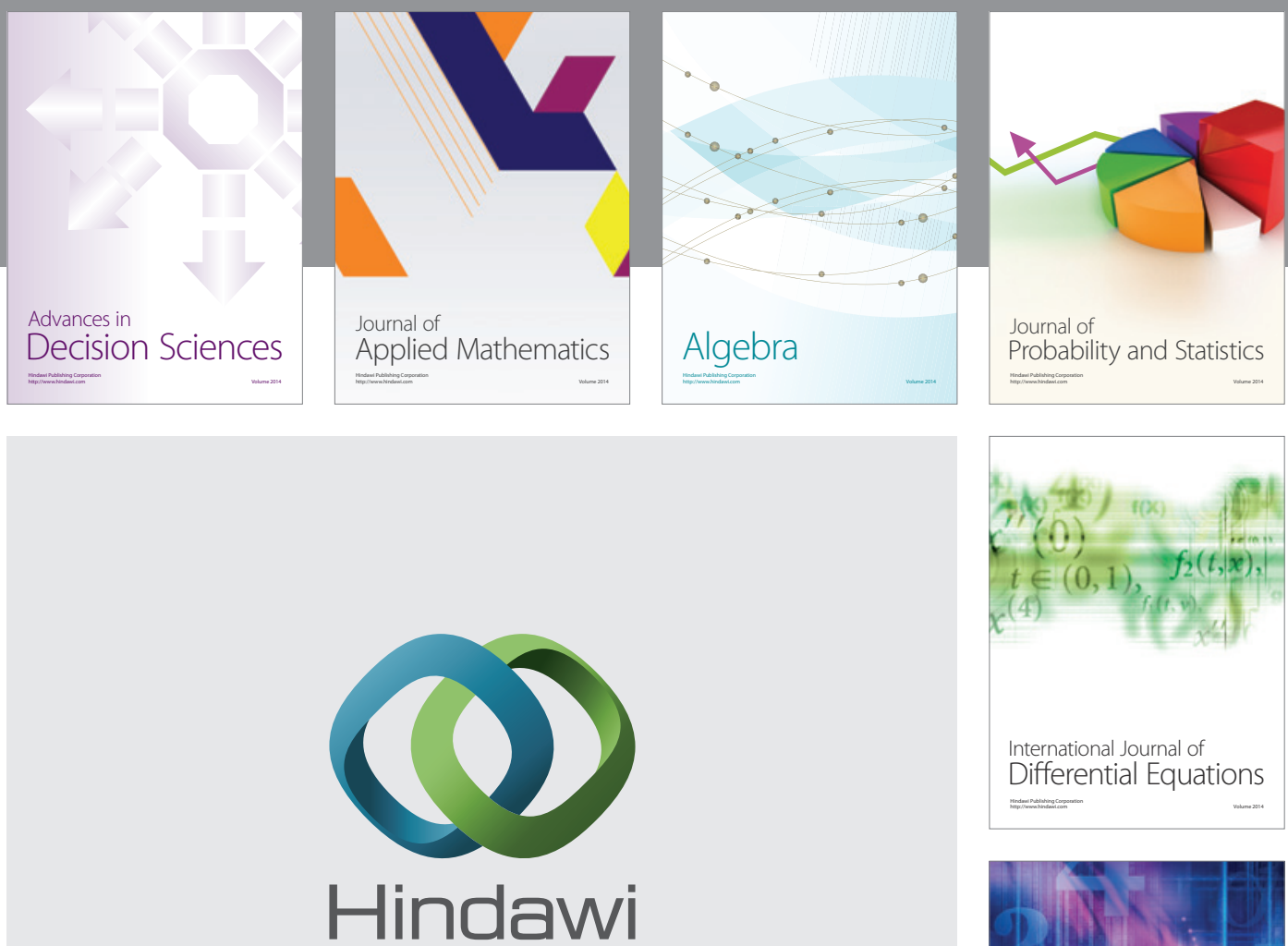

Submit your manuscripts at http://www.hindawi.com
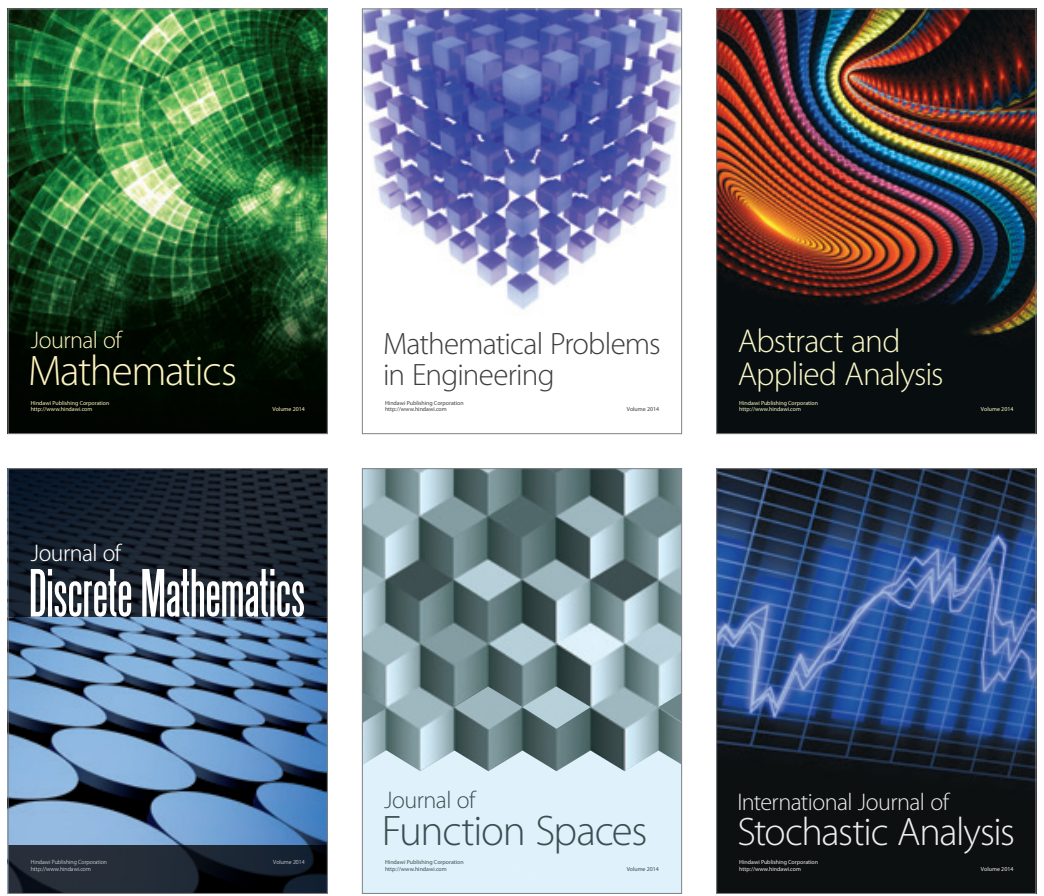

Journal of

Function Spaces

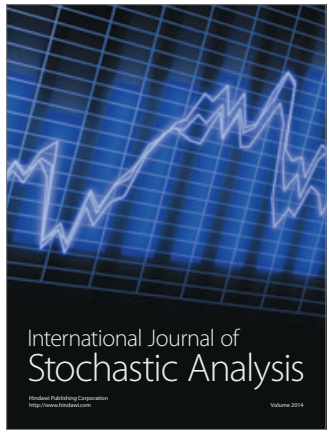

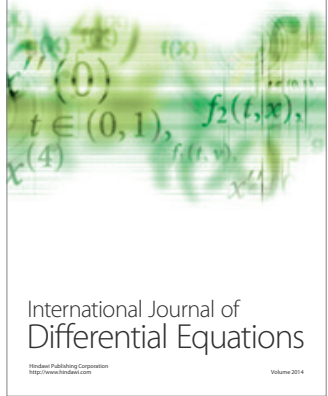
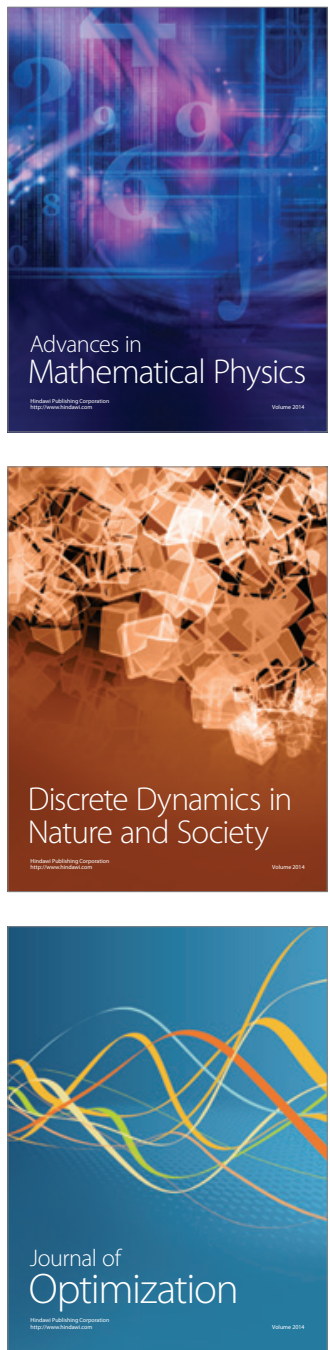